\begin{tabular}{|c|c|c|}
\hline \begin{tabular}{|l|} 
PROD. TYPE: COM \\
PP:1-11 (col.fig.: nil) \\
\end{tabular} & OPERES4808 DTD VER: 5.0.1 & $\begin{array}{l}\text { ED: Prathiba } \\
\text { PAGN: Vidya - SCAN: v4soft }\end{array}$ \\
\hline \multicolumn{3}{|c|}{ ARTICLE IN PRESS } \\
\hline 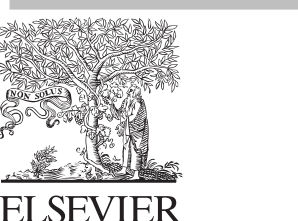 & $\begin{array}{l}\text { Available online at www.sciencedirect.com } \\
\text { SCIENCE d DiRECT }\end{array}$ & $\begin{array}{l}\text { Operations } \\
\text { Research } \\
\text { Letters } \\
\end{array}$ \\
\hline
\end{tabular}

www.elsevier.com/locate/dsw

1

\title{
On the exponential cardinality of FDS for the ordered $p$-median problem ${ }^{\hbar 2}$
}

\author{
J. Puerto ${ }^{\mathrm{a}, *}$, A.M. Rodríguez-Chía ${ }^{\mathrm{b}}$ \\ ${ }^{a}$ Dept. Estadistica e Investigacion Operativa, Facultad de Matemáticas, Universidad de Sevilla, 41012 Sevilla, Spain \\ ${ }^{\mathrm{b}}$ Facultad de Ciencias, Universidad de Cádiz, Spain
}

Received 4 June 2003; accepted 1 November 2004

\begin{abstract}
We study finite dominating sets (FDS) for the ordered median problem. This kind of problems allows to deal simultaneously with a large number of models. We show that there is no valid polynomial size FDS for the general multifacility version of

11 this problem even on path networks.
\end{abstract}

(c) 2004 Elsevier B.V. All rights reserved.

\section{Introduction}

Network location models have been widely studied in the literature as can be seen in several textbooks $[1,4,8,12]$. Since the seminal paper by Hakimi [7], much of this work has been devoted to identify finite sets of points where an optimal solution of a problem must belong to. These sets, called finite dominating sets (FDS), reduce the search for an optimal solution of a problem to a finite set of candidates.

\footnotetext{
The research of the authors is partially financed by Spanish research Grants BFM2001-2378, BFM2001-4028, BFM2004-0909 and HA2003-0121.

* Corresponding author. Tel.: +3495 4557940; fax: +34954622800 .

E-mail addresses: puerto@us.es (J. Puerto), antonio.rodriguezchia@uca.es (A.M. Rodríguez-Chía).
}

In the last years, a new type of function has attracted the attention of locators: the ordered median objective function. The corresponding ordered median problem allows a common algebraic analysis for a wide range of location models since many of the classical problems in location theory can be formulated as some of its particular instances. In the literature of location analysis, we can find a number of results concerning the ordered median problem, for example in the continuous case, characterizations of the optimal solution set and some algorithms have been obtained in [5,6,14-18]. On networks, finite dominating sets are known for particular instances of the problem, see $[10,11,13,19]$. Recently, also the discrete version of this model has been studied in [2,3]. (This objective function was already introduced in [21] in the context of multi-criteria decision making.) 
Nevertheless, it has been an open problem whether polynomial size FDS exist for the general version of this problem even on path networks. In this paper, we show that such sets do not exist.

An overview of the literature, involving characterizations of FDS, shows a lot of papers that succeed in finding this type of sets for different versions of location models. Ref. [9] is an excellent paper on this subject that characterizes FDS for a large number of location problems. However, we are not aware of any paper that states a negative result concerning existence of a polynomial size FDS for a given problem.

The main result in this paper proves that there exists a path graph with $n$ nodes satisfying the following property: there is a family of $O\left(n^{n}\right)$ ordered $\frac{n}{4}$-median problems defined on the above path graph, such that: (1) each problem has a unique optimal solution and (2) each optimal solution contains an element (facility) not included in any other solution. Therefore, in general, the multifacility ordered median problem cannot have polynomial size FDS.

To introduce the problem formally, some notation is needed. Let $G=(V, E)$ denote a path graph where $V=$ $\left\{v_{1}, \ldots, v_{n}\right\}$ is the set of nodes (demand points) and $E$ the set of edges. Suppose without loss of generality that the nodes are points on the real line, satisfying $v_{1} \leqslant \ldots \leqslant v_{n}$. Therefore, we denote by $\left[v_{i}, v_{i}+1\right]$ the edge that joins the nodes $v_{i}$ and $v_{i}+1$ for $i=$ $1, \ldots, n-1$. Let $A(G)$ be the interval $\left[v_{1}, v_{n}\right]$, then the distance from two points $x$ and $y$ in $A(G)$ is simply $d(x, y)=|x-y|$. In the same way, the distance from a node to a set with $p$ points, $X_{p}=\left\{x_{1}, \ldots, x_{p}\right\} \subseteq$ $A(G)$, is defined as

$d\left(v, X_{p}\right)=\min _{i=1, \ldots, p} d\left(v, x_{i}\right)=\min _{i=1, \ldots, p}\left|x_{i}-v\right|$.

Notice that $A(G)$ is a metric space which distance function is induced by the edge lengths, see [20].

We consider a set of non-negative weights $\left\{w_{1}, \ldots, w_{n}\right\}$, called $w$-weights, where the weight $w_{i}$ is associated to the node $v_{i}$ and represents the intensity of the demand at this node, for $i=1, \ldots, n$.

Let $\beta$ be a permutation of the set $\{1, \ldots, n\}$ satisfying that

$$
\begin{aligned}
w_{\beta_{1}} d\left(v_{\beta_{1}}, X_{p}\right) & \leqslant w_{\beta_{2}} d\left(v_{\beta_{2}}, X_{p}\right) \\
& \leqslant \ldots \leqslant w_{\beta_{n}} d\left(v_{\beta_{n}}, X_{p}\right) .
\end{aligned}
$$

For a given $\lambda=\left(\lambda_{1}, \ldots, \lambda_{n}\right)$, vector of non-negative components, called $\lambda$-weights, the $p$-facility ordered median function (the ordered $p$-median function) on $G$ is defined as

$$
\begin{aligned}
F_{\lambda}\left(X_{p}\right): & =\sum_{i=1}^{n} \lambda_{i} w_{\beta_{i}} d\left(v_{\beta_{i}}, X_{p}\right) \\
: & =\sum_{i=1}^{n} \lambda_{\sigma_{i}} w_{i} d\left(v_{i}, X_{p}\right),
\end{aligned}
$$

where $\sigma$ is a permutation of $\{1, \ldots, n\}$ such that $\sigma_{j}<\sigma_{k}$ if $w_{j} d\left(v_{j}, X_{p}\right) \leqslant w_{k} d\left(v_{k}, X_{p}\right)$ for all $j, k \in$ $\{1, \ldots, n\}$.

The $\lambda$-weights are the parameters that define the objective function and depending on the values of these parameters we obtain different problems. In fact, the ordered $p$-median problem allows to model the $p$ facility versions of the median $\left(\lambda_{i}=1, \forall i\right)$, center $\left(\lambda_{n}=1, \lambda_{i}=0, \forall i \neq n\right), \alpha$-centdian $\left(\lambda_{n}=1, \lambda_{i}=\right.$ $\alpha, \forall i \neq n), k$-centrum $\left(\lambda_{i}=1\right.$, for $i=n-k+$ $1, \ldots, n$ and $\lambda_{i}=0$ for $\left.i=1, \ldots, n-k\right), k$-trimmed $p$-mean location model (we omit the $\frac{k}{2}$ smallest and $\frac{k}{2}$ largest weighted distances, to simplify assume $k$ is even, $\lambda_{1}=\ldots=\lambda_{\frac{k}{2}}=0, \lambda_{\frac{k}{2}+1}=\ldots=\lambda_{n-\frac{k}{2}}=1$, $\lambda_{n-\frac{k}{2}+1}=\ldots=\lambda_{n}=0$ ), etc. Notice that we do not impose any assumption on the monotonicity of the $\lambda$ weights, therefore we do not restrict to the convex nor the concave cases, see [13].

Although we have already used the concept of FDS, in what follows, we give its formal definition.

Definition 1.1. Let $G=(V, E)$ be a graph with $n$ nodes and positive edge lengths. Let $w_{1}, \ldots, w_{n}$ be non-negative reals and $\lambda=\left(\lambda_{1}, \ldots, \lambda_{n}\right)$ a vector of non-negative components. A finite subset $X$ of $A(G)$ is an FDS, for the multifacility ordered median problem, if for any integer $p$ and $w$-weights associated to $v_{i}$ for $i=1, \ldots, n$, either $w_{i}$ or 0 there is an optimal solution, $X_{p}$, of the respective ordered $p$-median problem, such that $X_{p} \subset X$.

Obtaining an FDS for this model allows the development of different types of algorithms to solve it. Therefore, recently much effort has been devoted to obtain FDS for the ordered median problems (see $[10,11,13,19])$. In the following, we recall several 
1 sets used to define FDS for particular instances of the problem.

A point $x \in A(G)$ is in equilibrium with respect to the nodes $v_{k}, v_{l}, v_{k} \neq v_{l}$, if: $w_{k} d\left(v_{k}, x\right)=w_{l} d\left(v_{l}, x\right)$.

5 It is important to realize that there may exist subedges in equilibrium with respect to two nodes. We denote by

$7 E Q$ the set consisting of the nodes of $G$, the points in equilibrium which are isolated and the extreme points

9 of the subedges in equilibrium. Moreover, we consider the following sets:

$$
\begin{aligned}
Y= & \left\{y \in A(G): w_{i} d\left(v_{i}, y\right)=w_{j} d\left(v_{j}, z\right),\right. \\
& \left.v_{i}, v_{j} \in V, z \in E Q\right\}, \\
T= & \left\{X_{2}=\left(x_{1}, x_{2}\right) \in A(G) \times A(G): \exists v_{r}, v_{s}\right.
\end{aligned}
$$
served by $x_{1}$ and $v_{r^{\prime}}, v_{s^{\prime}}$ served by $x_{2}$, such that $w_{r} d\left(v_{r}, x_{1}\right)=w_{r^{\prime}} d\left(v_{r^{\prime}}, x_{2}\right)$ and $w_{s}$ $d\left(v_{s}, x_{1}\right)=w_{s^{\prime}} d\left(v_{s^{\prime}}, x_{2}\right)$. Moreover, if $w_{r}=w_{r^{\prime}}$ and $w_{s}=w_{s^{\prime}}$, then the slopes of the functions $d\left(v_{r}, \cdot\right)$ and $d\left(v_{s}, \cdot\right)$, in the edge that $x_{1}$ belongs to, must have the same signs at $x_{1}$ and the slopes of the functions $d\left(v_{r^{\prime}}, \cdot\right)$ and $d\left(v_{s^{\prime}}, \cdot\right)$, in the edge that $x_{2}$ belongs to, must have different signs at $x_{2}$.

Ref. [13] proves that for $\lambda_{1} \geqslant \ldots \geqslant \lambda_{n} \geqslant 0$ the node set $V$ constitutes an FDS for the multifacility ordered median problem. For arbitrary non-negative $\lambda$-weights, it also obtains that $E Q$ is an FDS for the single-facility ordered median problem.

Ref. [11] studies the multifacility ordered median problem where the $\lambda$-weights are defined as

$a=\lambda_{1}=\ldots=\lambda_{k} \neq \lambda_{k+1}=\ldots=\lambda_{n}=b$,

for a fixed $k$, such that, $1 \leqslant k<n$. It proves that the set $Y$ is an FDS for this problem.

Ref. [19] proves that the set $F=(E Q \times Y) \cup T \subset$ $A(G) \times A(G)$ contains an optimal solution of the ordered 2-median problem in any network for any choice of $\lambda$-weights.

Ref. [10] gives an FDS for the single facility ordered median problem with general node weights (the $w$-weights can be negative). Moreover, for the case of a directed network with non-negative $w$-weights, it proves that there is always an optimal solution in $V$.

However, none of these papers deals with the general case of the multifacility ordered median problem.
In fact, these papers impose very restrictive hypotheses such that their respective results cannot be extended any further. Indeed, only [13] and [11] consider $p$-facility problems for any $p>2$, although for particular cases: [13] when the $\lambda$-weights are given in non increasing order and [11] when the $\lambda$-weights satisfy $a=\lambda_{1}=\ldots=\lambda_{k} \neq \lambda_{k+1}=\ldots=\lambda_{n}=b$, for some $k, 1 \leqslant k<n$.

\section{On the exponential cardinality of FDS for the $p$-facility ordered median problem}

In this section we prove that there is no polynomial size FDS for the general ordered $p$-median problem even on path networks. In order to do that we consider a path graph $G$ where $V=\left\{v_{1}, \ldots, v_{2}\right\}$, being $p$ a fixed natural number, $v_{1}=0, v_{2}=2, v_{2 i-1}=v_{2 i-2}+M$ and $v_{2 i}=v_{2 i-1}+2^{i}$, for $i=2, \ldots, p$, and $M=4 \sum_{i=1}^{p} 2^{i}+1$ (a sufficiently large number) (see Fig. 1).

The $w$-weights associated to the nodes are assumed to be equal to one and the $\lambda$-weights are given as follows:

$\lambda_{1}=0, \lambda_{2}=\lambda_{3}=2 p, \lambda_{4}=p \quad$ and

$\lambda_{i}=\frac{2^{2 p}+1}{2^{2 p+1}}\left(\lambda_{i-2}+\lambda_{i-1}\right)$,

for $i=5, \ldots, 2 p$.

Under these conditions our goal is to find $p$ points on $A(G), X_{p}=\left\{x_{1}, \ldots, x_{p}\right\}$, solving the following problem:

$\min _{X_{p} \subseteq A(G)} F\left(X_{p}\right):=\sum_{i=1}^{2 p} \lambda_{\sigma_{i}} d\left(v_{i}, X_{p}\right)$,

where $\sigma$ is a permutation of $\{1, \ldots, 2 p\}$, such that, $\sigma_{k}<\sigma_{l}$ if $d\left(v_{k}, X_{p}\right) \leqslant d\left(v_{l}, X_{p}\right)$ for each $k, l \in$ $\{1, \ldots, 2 p\}$. (In this case, we say that the $\lambda$-weight $\lambda_{\sigma_{i}}$ is assigned (allocated) to the node $v_{i}$.)

Remark 2.1. Notice that, the $\lambda$-weights defined in (2) satisfy the relationships:

$$
\begin{aligned}
& 2 \max \left\{\lambda_{i-2}, \lambda_{i-1}\right\}>2 \lambda_{i}>\lambda_{i-2}+\lambda_{i-1}, \\
& \quad \text { for all } i=5, \ldots, 2 p, \\
& 2 \lambda_{4}=\lambda_{2}=\lambda_{3}>\lambda_{1}=0, \\
& 2 \lambda_{2}>\lambda_{4}+\lambda_{5}+\lambda_{8} .
\end{aligned}
$$




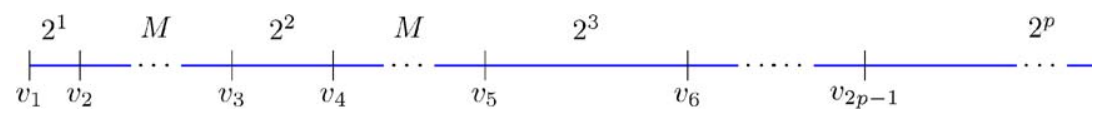

Fig. 1. Illustration of the graph in Section 2.

1 Moreover, the components of the vector $\lambda=$ $\left(\lambda_{1}, \ldots, \lambda_{2 p}\right)$ satisfy the following chain of inequali3 ties:

$$
\begin{aligned}
\lambda_{2} & =\lambda_{3}>\ldots>\lambda_{2 p-3}>\lambda_{2 p-1}>\lambda_{2 p}>\lambda_{2 p-2} \\
& >\lambda_{2 p-4}>\ldots>\lambda_{4}>\lambda_{1}=0 .
\end{aligned}
$$

Therefore

(i) $\lambda_{2 j+1}>\lambda_{2(j+1)+1}, \quad$ for $j=1, \ldots, p-2$ (the sequence of $\lambda$-weights with odd indexes $(>1)$ is decreasing).

(ii) $\lambda_{2(j+1)}>\lambda_{2 j}$, for $j=2, \ldots, p-1$ (the sequence of $\lambda$-weights with even indexes $(>2)$ is increasing).

(iii) $\lambda_{2 j-1}>\lambda_{2 i}, \quad$ for any $i, j \in\{2, \ldots, p\}$ (a $\lambda$ weight with odd index $(>1)$ is always greater than any other with an even index $(>2))$.

(iv) $\lambda_{2 j}<\lambda_{k}$, if $k>2 j, j>1$ and $\lambda_{2 j+1}>\lambda_{k}$, if $k>2 j+1, j \geqslant 1$.

We will prove that the optimal policy to solve Problem (3) is to locate a service facility on each edge $\left[v_{2 i-1}, v_{2 i}\right]$ for $i=1, \ldots, p$ and to assign $\lambda_{i}$ to $v_{i}$ for $i=1, \ldots, 2 p$.

Lemma 2.1. If $X_{p}=\left\{x_{1}, \ldots, x_{p}\right\}$ is an optimal solution of Problem (3) then $x_{i} \in\left[v_{2 i-1}, v_{2 i}\right]$ for $i=$ $1, \ldots, p$.

Proof. First, we prove that the nodes $v_{2 i}$ and $v_{2 i+1}$ for $i=1, \ldots, p$, are not covered by the same service facility. Suppose on the contrary that there exists $j \in$ $\{1, \ldots, p\}$, such that, $v_{2 j}$ and $v_{2 j+1}$ are served by the same service facility $x \in X_{p}$. This implies that the following terms would appear in the objective function: $\lambda_{\sigma_{2 j}} d\left(v_{2 j}, x\right)+\lambda_{\sigma_{2 j+1}} d\left(v_{2 j+1}, x\right)$.

Notice that $d\left(v_{2 j}, x\right)+d\left(v_{2 j+1}, x\right) \geqslant M$. Moreover

1. If both $\sigma_{2 j}$ and $\sigma_{2 j+1}$ are different from 1, we have that $\lambda_{\sigma_{2 j}} \geqslant \lambda_{4}, \lambda_{\sigma_{2 j+1}} \geqslant \lambda_{4}$, and at least one of these inequalities is strict.
2. If $\sigma_{2 j}=1$, then $d\left(v_{2 j+1}, x\right) \geqslant M / 2$. In a similar way, the case $\sigma_{2 j+1}=1$ implies that $d\left(v_{2 j}, x\right) \geqslant M / 2$. In all cases

$$
\begin{aligned}
& \lambda_{\sigma_{2 j}} d\left(v_{2 j}, x\right)+\lambda_{\sigma_{2 j+1}} d\left(v_{2 j+1}, x\right)>\frac{M-1}{2} \lambda_{4} \\
& \quad=2 \lambda_{4} \sum_{i=1}^{p} 2^{i}=\lambda_{2} \sum_{i=1}^{p} 2^{i} .
\end{aligned}
$$

The inequality above contradicts the optimality of $X_{p}$. Indeed, consider $X_{p}^{\prime}=\left\{x_{1}^{\prime}, \ldots, x_{p}^{\prime}\right\}$ such that $x_{i}^{\prime}$ is located at the midpoint of the edge $\left[v_{2 i-1}, v_{2 i}\right]$ for $i=1, \ldots, p$. Then, since $\lambda_{2} \geqslant \lambda_{i}$ for $i=1, \ldots, 2 p$, we have that $F\left(X_{p}^{\prime}\right) \leqslant \lambda_{2} \sum_{i=1}^{p} 2^{i}$. Therefore, $v_{2 i}$ and $v_{2 i+1}$ for $i=1, \ldots, p$, can not be covered by the same service facility.

Hence, in what follows, we can assume without loss of generality that each service facility $x_{i}$ covers the demand of $v_{2 i-1}$ and $v_{2 i}$ for $i=1, \ldots, p$.

The fact that $x_{i} \in\left[v_{2 i-1}, v_{2 i}\right]$ follows directly from the isotonicity property of the ordered median objective with nonnegative $\lambda$-weights (see Theorem 1 in [5]). Indeed, if $x_{i} \notin\left[v_{2 i-1}, v_{2 i}\right]$ for some $i$, $i=1, \ldots, p$, we move $x_{i}$ to its closest node in the interval $\left[v_{2 i-1}, v_{2 i}\right]$, the new vector of distances of $\left\{v_{1}, \ldots, v_{2 p}\right\}$ from the servers is smaller than the old vector.

Remark 2.2. Since all the $w$-weights are equal to one, by symmetry arguments and without loss of generality, in what follows we only consider solutions of this problem satisfying that $d\left(v_{2 i-1}, x_{i}\right) \leqslant d\left(v_{2 i}, x_{i}\right)$ for $i=1, \ldots, p$, and consequently, by the structure of the graph, $d\left(v_{2 i}, x_{i}\right) \leqslant d\left(v_{2 i+2}, x_{i+1}\right)$ for $i=1, \ldots, p-1$. Hence,

(i) $\sigma_{2 i-1}<\sigma_{2 i}$ for $i=1, \ldots, p$,

(ii) $\sigma_{2 i}<\sigma_{2 i+2}$ for $i=1, \ldots, p-1$.

The above assertions imply that $\sigma_{2 p}=2 p$. Moreover, by Lemma 2.1 and for the sake of the readability, we can represent the graph of Fig. 1 as a graph with only 


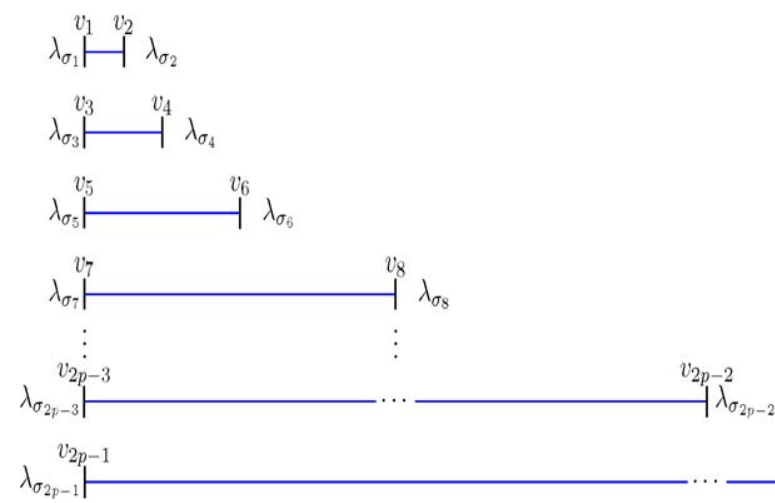

Fig. 2. The new representation of the graph where $\sigma_{2 i}>\sigma_{2 i-1}$, for $i=1, \ldots, p$ and $\sigma_{2 i+2}>\sigma_{2 i}$, for $i=1, \ldots, p-1$.

$1 \quad p$ edges where the edges with length $M$ are omitted (see Fig. 2).

3 Theorem 2.1. If $X_{p}$ is an optimal solution of Problem (3) then $\lambda_{\sigma_{i}}=\lambda_{i}$ for $i=1, \ldots, 2 p$.

5 Proof. First, we prove that $\lambda_{1}$ must be assigned to $v_{2 i-1}$ for some $i, i=1, \ldots, p$. Indeed, if $\sigma_{2 i}=1$

7 for some $i, i=1, \ldots, p$ then, by Remark 2.2.i, $\sigma_{2 i-1}<1$. However, this is impossible because $9 \sigma_{2 i-1} \in\{1, \ldots, 2 p\}$.

Second, by Lemmas A.1 and A.2, for $k=1, \ldots, p$, we have that $\lambda_{2 k}$ is assigned to $v_{2 i}$ for some $i, i=$ $1, \ldots, p$. Moreover, Remark 2.2(ii), implies that $\sigma_{2 i}=$ $2 i$, (i.e. $\lambda_{2 i}$ is assigned to $v_{2 i}$ ) for $i=1, \ldots, p$. Therefore, using a recursive argument and Remark 2.2(i), we obtain that $\sigma_{1}=1$ (i.e. $\lambda_{1}$ is assigned to $v_{1}$ ), $\sigma_{3}=3$ and so on. Thus, the result follows.

Remark 2.3. The above result has been proven assuming that $\left|v_{2 i+1}-v_{2 i+2}\right|=2\left|v_{2 i-1}-v_{2 i}\right|$. However, the reader may notice that the result also holds whenever $\left|v_{2 i+1}-v_{2 i+2}\right| \geqslant 2\left|v_{2 i-1}-v_{2 i}\right|$.

In order to disprove the polynomial cardinality of any FDS for the multifacility ordered me1 (assume that $p$ is even). Let $P=\{1, \ldots, p\}$ and $J=\left\{j_{1}, j_{2}, \ldots, j_{\frac{p}{2}-1}, j_{\frac{p}{2}}\right\} \subseteq P$, such that, $1=j_{1}<j_{2}<\ldots<j \frac{p}{2}-1<j \frac{p}{2}=p$. On the graph $G$ we formulate the following $\left(\frac{p}{2}\right)$-facility ordered median problem:

$\min _{X \frac{p}{2} \subseteq A(G)} \sum_{i=1}^{2 p} \lambda_{\sigma_{i}}^{\prime} w_{i}^{\prime} d\left(v_{i}, X_{\frac{p}{2}}\right)$,

where $\lambda^{\prime}=\left(0, \ldots, 0, \lambda_{1}, \ldots, \lambda_{p}\right)$, such that, $\lambda_{i}$ is defined by (2) for $i=1, \ldots, p, w_{2 j-1}^{\prime}=w_{2 j}^{\prime}=1$ for each $j \in J$ and $w_{2 j-1}^{\prime}=w_{2 j}^{\prime}=0$ for each $j \in P \backslash J$.

Moreover, since $w_{2 j-1}^{\prime} d\left(v_{2 j-1}, X_{\frac{p}{2}}\right)=w_{2 j}^{\prime} d\left(v_{2 j}\right.$, $\left.X_{\frac{p}{2}}\right)=0 \forall j \in P \backslash J$, the first $p$ positions of the ordered sequence of weighted distances between each node and its service facility are given by $w_{2 j-1}^{\prime} d\left(v_{2 j-1}, X_{\frac{p}{2}}\right), w_{2 j}^{\prime} d\left(v_{2 j}, X_{\frac{p}{2}}\right)$ with $j \in P \backslash J$. (Indeed, these positions are always zeros.) Thus, we can assume without loss of generality that the $\lambda$ weights allocated to $v_{2 j-1}$ and $v_{2 j}$ for any $j \in P \backslash J$ are the first $p$ components of the vector $\lambda^{\prime}$, that is, 0 .

Notice that the nodes $v_{2 j-1}$ and $v_{2 j} \forall j \in P \backslash J$ are not really taken into account in the objective value because $w_{2 j-1}^{\prime}=w_{2 j}^{\prime}=0$. Thus, using Lemma 2.1, this problem reduces to locate $p / 2$ service facilities on a graph with $p / 2$ edges. Indeed, if we consider

$V^{\prime}=\bigcup_{i=1, j_{i} \in J}^{\frac{p}{2}}\left\{v_{2 j_{i}-1}, v_{2 j_{i}}\right\}:=\bigcup_{i=1}^{\frac{p}{2}}\left\{v_{2 i-1}^{\prime}, v_{2 i}^{\prime}\right\}$

and the path graph $G^{\prime}$ induced by the set of nodes $V^{\prime}$, Problem (7) can be reformulated as

$\min _{X_{\frac{p}{2}} \subseteq A\left(G^{\prime}\right)} \sum_{i=1}^{p} \lambda_{\sigma_{i}} d\left(v_{i}^{\prime}, X_{\frac{p}{2}}\right)$. 
1 Observe that the components of the vector $\lambda=$ $\left(\lambda_{1}, \ldots, \lambda_{p}\right)$ coincide with the first $p$ entries of (2)

3 and therefore they satisfy (4)-(6). In addition, $\sigma_{i} \in$ $\{1, \ldots, p\}$ and $\sigma_{i}<\sigma_{j}$ if $d\left(v_{i}^{\prime}, X_{\frac{p}{2}}\right) \leqslant d\left(v_{j}^{\prime}, X_{\frac{p}{2}}\right)$ (the 5 $w$-weights are all equal to one).

Theorem 2.2. If $X_{p / 2}$ is the optimal solution of Prob7 lem (7) then $x_{i}=v_{2 j_{i}-1}+z_{i}$ with $z_{1}=1$ and $z_{i}=$ $2^{j_{i-1}}-z_{i-1}$ for $i=2, \ldots, p / 2$.

9 Proof. To prove this result we consider the equivalent formulation of Problem (7) given in (8). Applying

11 Theorem 2.1 and Remark 2.3, we get that the $\lambda$-weight allocated to the node $v_{i}^{\prime}$ is $\lambda_{i}$ for $i=1, \ldots, p$.

In addition, the solution $X_{\frac{p}{2}}$ satisfies the relation$\operatorname{ship} d\left(v_{2 i}^{\prime}, x_{i}\right)=d\left(v_{2 i+1}^{\prime}, x_{i+1}\right)$ for $i=1, \ldots, p / 2-1$. Indeed, since $\lambda_{2 i}$ and $\lambda_{2 i+1}$ are assigned to $v_{2 i}^{\prime}$ and $v_{2 i+1}^{\prime}$, respectively, for $i=1, \ldots, p / 2-1$, then $d\left(v_{2 i}^{\prime}, x_{i}\right) \leqslant d\left(v_{2 i+1}^{\prime}, x_{i+1}\right)$. Moreover, $x_{i+1}$ must be located as close as possible to $v_{2 i+1}^{\prime}$ because $\lambda_{2 i+1}>\lambda_{2 i+2}$, which in turn implies that $d\left(v_{2 i}^{\prime}, x_{i}\right)=d\left(v_{2 i+1}^{\prime}, x_{i+1}\right)$.

Next, we prove that $d\left(v_{1}^{\prime}, x_{1}\right)=1$. Notice that, by Remark 2.2, we have that $d\left(v_{1}^{\prime}, x_{1}\right) \leqslant 1$. If $d\left(v_{1}^{\prime}, x_{1}\right)<1$ then we would move $x_{1}$ towards $v_{2}^{\prime}$ a small enough amount, $\xi$. This movement would allow us to move $x_{i}$ towards $v_{2 i-1}^{\prime}$ for any even index $i=2, \ldots, p / 2$, and $x_{j}$ towards $v_{2 j}^{\prime}$ for any odd in$\operatorname{dex} j=2, \ldots, p / 2$ by the same amount $\xi$; without any reassignment of the $\lambda$-weights. These movements would produce the following change in the objective function:

$\xi\left(-\left(\lambda_{2}-\lambda_{1}\right)+\sum_{k=2}^{\frac{p}{2}}(-1)^{k-1}\left(\lambda_{2 k-1}-\lambda_{2 k}\right)\right)$.

This amount is negative because $-\left(\lambda_{2}-\lambda_{1}\right)$ is negative and $\left\{\lambda_{2 k-1}-\lambda_{2 k}\right\}_{k \geqslant 2}$ is a decreasing sequence of positive values, that is, $\lambda_{2 k-1}-\lambda_{2 k}>\lambda_{2 k+1}-\lambda_{2 k+2}>0$ for $k=1, \ldots, p / 2$. However, this is not possible since $X_{\frac{p}{2}}$ is optimal. Therefore, we obtain that $x_{1}=v_{1}^{\prime}+$ 1 and that $X_{\frac{p}{2}}$ is the unique solution satisfying that $d\left(v_{2 i-1}, x_{i}\right) \leqslant d\left(v_{2 i}, x_{i}\right)$ for $i=1, \ldots, p$. (See Remark 2.2.) Finally, since $d\left(v_{2 i}^{\prime}, x_{i}\right)=d\left(v_{2 i+1}^{\prime}, x_{i+1}\right)$ for $i=$ $1, \ldots, p / 2-1$, the result follows.
Our next result proves that there is no polynomial size cardinality FDS for the multifacility ordered median problem. The proof consists of building a family of $O\left(n^{n}\right)$ problems on the same graph with different solutions (each solution contains at least one point not included in the remaining), $n$ being the number of nodes.

Theorem 2.3. There is no polynomial size FDS for the multifacility ordered median problem.

Proof. Consider Problem (7), by Theorem 2.2 and Remark 2.2, for each choice of the set $J \subseteq P$, we have an unique optimal solution satisfying that $d\left(v_{2 i-1}, x_{i}\right) \leqslant d\left(v_{2 i}, x_{i}\right)$ for $i=1, \ldots, p$, such that, the service facility located on the edge $\left[v_{2 p-1}, v_{2 p}\right]$ has a different location, recall that $j_{p / 2}=p$. Thus, since there are $\left(\begin{array}{c}2 p-2 \\ \frac{p}{2}-2\end{array}\right)$ different choices of the set $J$, any FDS for the considered problem contains at least $\left(\begin{array}{c}2 p-2 \\ \frac{p}{2}-2\end{array}\right)$ elements.

Therefore, we have found a family of problems for which a valid FDS is at least of order $O\left(n^{n}\right)$ where $n$ denotes the number of nodes (recall that in our case $n=2 p$ ).

Remark 2.4. Problem (7) is formulated based on the concrete $\lambda$ given in (2). Nevertheless, a detailed reading of the proofs shows that any $\lambda$ satisfying (4)-(6) would be also valid.

\section{Concluding remarks}

This paper proves that polynomial size FDS cannot exist for the multifacility ordered median problem. However, it is still an open question whether polynomial size FDS may exist for the convex version of this problem ( $\lambda$-weights given in non-decreasing order).

\section{Acknowledgements}

The authors would like to thank professor Arie Tamir for his valuable comments on an earlier version of this paper, as well as to an anonymous referee for his careful reading of the manuscript. 


\section{Appendix A.}

3 Lemma A.1. If $X_{p}$ is an optimal solution of Problem (3) then for $k=2, \ldots, p, \lambda_{2 k}$ is assigned to $v_{2 i}$ for 5 some $i, i=1, \ldots, p$.

Proof. Suppose on the contrary that $\lambda_{2 k}$ is assigned to $v_{2 j-1}$ for some $j, j=1, \ldots, p$. We can assume without loss of generality that $2 k$ is the maximum $1, \ldots, p$. with $j=1, \ldots, p$. Recall that $k$ must be less than $p$ since $\sigma_{2 p}=2 p$ (see Remark 2.2). In what follows we distinguish two cases depending on the type of node where $\lambda_{2 k+1}$ has been assigned to.

Case 1: $\lambda_{2 k+1}$ is assigned to $v_{2 j^{\prime}}$ for some $j^{\prime}, j^{\prime}=$

By Remark 2.2(i) we have that $\sigma_{2 j}>\sigma_{2 j-1}=2 k$.

$$
\xi\left(\lambda_{2 k}+\lambda_{2 k+1}-\lambda_{\sigma_{2 j}}-\lambda_{\sigma_{2 j^{\prime}}}\right) .
$$

Thus, by Remark 2.1(iv), we must have that $\lambda_{\sigma_{2 j}}>\lambda_{\sigma_{2 j-1}}=\lambda_{2 k}$. Hence, since $X_{p}$ is optimal, $x_{j}$ must be located as far as possible from $v_{2 j-1}$. Besides, since $\sigma_{2 j-1}=2 k<2 k+1=\sigma_{2 j^{\prime}}$ then $d\left(v_{2 j-1}, x_{j}\right) \leqslant d\left(v_{2 j^{\prime}}, x_{j^{\prime}}\right)$. Therefore, we have that $d\left(v_{2 j-1}, x_{j}\right)=d\left(v_{2 j^{\prime}}, x_{j^{\prime}}\right)$ and we can reassign the $\lambda$-weights, so that $\lambda_{2 k}$ is assigned to $v_{2 j^{\prime}}$ and $\lambda_{2 k+1}$ to $v_{2 j-1}$.

Case 2: $\lambda_{2 k+1}$ is assigned to $v_{2 j^{\prime}-1}$ for some $j^{\prime}$, $j^{\prime}=1, \ldots, p$.

Assume that $d\left(v_{2 j-1}, x_{j}\right) \neq d\left(v_{2 j}, x_{j}\right)$ and $d\left(v_{2 j^{\prime}-1}, x_{j^{\prime}}\right) \neq d\left(v_{2 j^{\prime}}, x_{j^{\prime}}\right)$. Under this assumption, we can move $x_{j}$ and $x_{j^{\prime}}$ towards $v_{2 j}$ and $v_{2 j^{\prime}}$, respectively, by the same small enough amount, $\xi$, without any reassignment of the $\lambda$-weights (see Fig. $3\left(\right.$ a)).This is possible because $2 k+2=\sigma_{2 j^{\prime \prime}}$ for some $j^{\prime \prime}, j^{\prime \prime}=1, \ldots, p$ (recallthat $\lambda_{2 k}$ is the maximum index of a $\lambda$-weight assigned to a node with odd index) and $d\left(v_{2 j^{\prime}-1}, x_{j^{\prime}}\right)$ as well as $d\left(v_{2 j-1}, x_{j}\right)$ are strictly smaller than $d\left(v_{2 j^{\prime \prime}}, x_{j^{\prime \prime}}\right)$. These movements imply the following change in the objective function:

This amount is negative. Indeed, since $\sigma_{2 j}>2 k+1$ and $\sigma_{2 j^{\prime}}>2 k+1$, by Remark 2.1(iv), we get $\lambda_{\sigma_{2 j}}+\lambda_{\sigma_{2 j^{\prime}}}>2 \lambda_{2 k+2}$ and, by (4), we have that $2 \lambda_{2 k+2}>\lambda_{2 k}+\lambda_{2 k+1}$. This is a contradiction because $X_{p}$ was an optimal solution.
In what follows, we study the cases $d\left(v_{2 j-1}, x_{j}\right)=$ $d\left(v_{2 j}, x_{j}\right)$ and $d\left(v_{2 j^{\prime}-1}, x_{j^{\prime}}\right)=d\left(v_{2 j^{\prime}}, x_{j^{\prime}}\right)$.

Case 2.1: $d\left(v_{2 j^{\prime}-1}, x_{j^{\prime}}\right)=d\left(v_{2 j^{\prime}}, x_{j^{\prime}}\right)$.

Since $\sigma_{2 j^{\prime}-1}=2 k+1$ we can assume without loss of generality that $\sigma_{2 j^{\prime}}=2 k+2$. Now, since $\lambda_{2 k+1}$ and $\lambda_{2 k+2}$ have been already assigned and, by Remark 2.2(i), $\sigma_{2 j}>2 k$ we get that $\sigma_{2 j}>2 k+2=\sigma_{2 j^{\prime}}$. This means, by Remark 2.2(ii), that $j>j^{\prime}$. Moreover, since $\sigma_{2 j}>2 k$ then, by Remark 2.1(iv), $\lambda_{\sigma_{2 j}}>\lambda_{\sigma_{2 j-1}}=\lambda_{2 k}$. Hence, $x_{j}$ must be located as far as possible from $v_{2 j-1}$ because $X_{p}$ is optimal. Besides, the relationship $\sigma_{2 j-1}=2 k<2 k+1=\sigma_{2 j^{\prime}-1}$ implies that $d\left(v_{2 j-1}, x_{j}\right) \leqslant d\left(v_{2 j^{\prime}-1}, x_{j^{\prime}}\right)$. Therefore, we obtain that $d\left(v_{2 j-1}, x_{j}\right)=d\left(v_{2 j^{\prime}-1}, x_{j^{\prime}}\right)$. This permits reassigning the $\lambda$-weights so that $\lambda_{2 k}$ is assigned to $v_{2 j^{\prime}-1}$, $\lambda_{2 k+1}$ to $v_{2 j^{\prime}}$ and $\lambda_{2 k+2}$ to $v_{2 j-1}$ (see Fig. 3(b)). However, this allocation induces a contradiction because $2 k$ is the maximum even index of a $\lambda$-weight assigned to a node with odd index.

Case 2.2: $d\left(v_{2 j-1}, x_{j}\right)=d\left(v_{2 j}, x_{j}\right)$. The analysis of this case is analogous to the Case 2.1 and also induces a contradiction.

After this case analysis, we conclude that the optimal assignment of the $\lambda$-weights satisfies that each $\lambda_{2 k}$ for any $k=2, \ldots, p$, is allocated to $v_{2 i}$ for some $i, i=1, \ldots, p$.

The result above describes the optimal assignment of the $\lambda$-weights with even index, $k>2$. However, it is still missing the case $\lambda_{2}$. The following result analyzes this case:

Lemma A.2. If $X_{p}$ is an optimal solution of Problem (3) then $\lambda_{2}$ must be assigned to $v_{2}$.

Proof. First, notice that if $\lambda_{2}$ were assigned to $v_{2 i}$ for some $i, i=1, \ldots, p$, then by Remark 2.2(ii) and since $\lambda_{1}$ is already assigned to a $v_{2 j-1}$ for some $j$, $j=1, \ldots, p$, we would have that $i=1$.

In order to prove the result, we proceed by contradiction assuming that $\lambda_{2}$ is assigned to $v_{2 j-1}$ for some $j, j=1, \ldots, p$. Therefore, since by Lemma A.1, for $k=2, \ldots, p, \lambda_{2 k}$ is assigned to $v_{2 i}$ for some $i$ with $i=1, \ldots, p$, and $\lambda_{2}$ is assigned to $v_{2 j-1}$ then there exists only one $j_{o} \in\{1, \ldots, p\}$ such that $\lambda_{2 j_{o}-1}$ is assigned to a node $v_{2 i}$ for some $i, i=1, \ldots, p$. Depending on the value of $j_{o}$, we distinguish the following 


\section{ARTICLE IN PRESS}

J. Puerto, A.M. Rodríguez-Chía / Operations Research Letters III (III) III-III

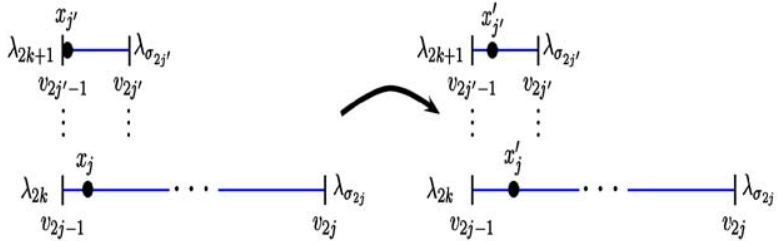

(a)

Case 2.

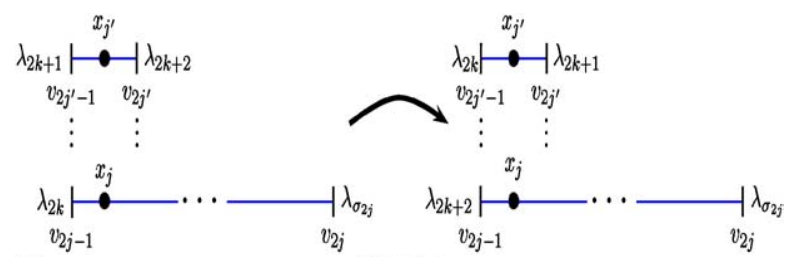

(b)

Fig. 3. Illustration of Lemma A.1.

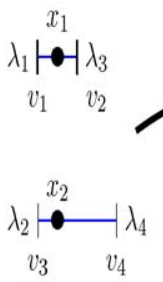

(a)
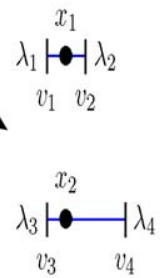

Case 1.

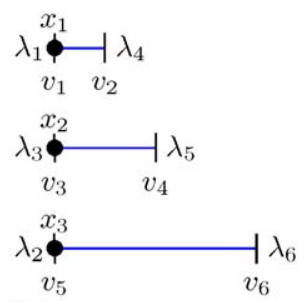

(b)

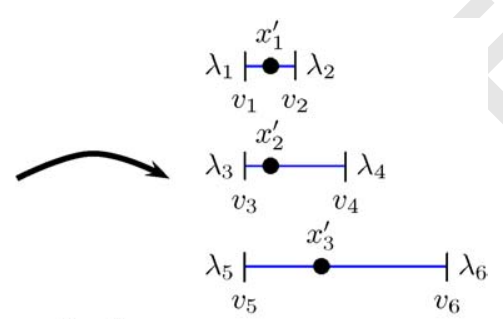

Case 2.

Fig. 4. Illustration of Lemma A.2.

cases:

Case 1: $j_{o}=2$ (see Fig. 4(a)). of the Lemma.
If $\lambda_{3}$ is assigned to $v_{2 i}$ for some $i, i=1, \ldots, p$, then, by Remark 2.2(ii) and Lemma A.1, $\lambda_{3}$ must be assigned to $v_{2}(i=1)$ and $\lambda_{4}$ to $v_{4}$. By Remark 2.2(i), $\sigma_{1}<3$ and $\sigma_{3}<4$ then $\sigma_{1} \leqslant 2$ and $\sigma_{3} \leqslant 2$. Therefore, $\lambda_{1}$ is assigned either to $v_{1}$ or $v_{3}$ and the same occurs with $\lambda_{2}$. In any case, to minimize the objective function we must have that $d\left(v_{1}, x_{1}\right)=d\left(v_{2}, x_{2}\right)=d\left(v_{3}, x_{3}\right)$ and this implies that we can reassign the $\lambda$-weights such that $\lambda_{1}$ goes to $v_{1}, \lambda_{2}$ to $v_{2}$ and $\lambda_{3}$ to $v_{3}$. Since the objective value does not change, we get the thesis

Case 2: $j_{o}=3$ (see Fig. 4(b)).

If $\lambda_{5}$ is assigned to $v_{2 i}$ for some $i, i=1, \ldots, p$, then, by Remark 2.2(ii) and Lemma A.1, $\lambda_{4}$ must be assigned to $v_{2}, \lambda_{5}$ to $v_{4}$ and $\lambda_{2 i}$ to $v_{2 i}$ for any $i=3, \ldots, p$. Since $\lambda_{4}, \lambda_{5}$ and $\lambda_{6}$ have been already allocated and Remark 2.2(i) ensures that $\sigma_{1}<4, \sigma_{2}<5$ and $\sigma_{3}<6$ then $\sigma_{1} \leqslant 3, \sigma_{3} \leqslant 3$ and $\sigma_{5} \leqslant 3$. Moreover

(i) Since $\lambda_{4}<\lambda_{5}$ then $d\left(v_{1}, x_{1}\right) \leqslant d\left(v_{3}, x_{2}\right)$. (Otherwise the objective function may decrease). Indeed, if $d\left(v_{1}, x_{1}\right)>d\left(v_{3}, x_{2}\right)$ we move $x_{1}$ and $x_{2}$ towards $v_{1}$ and $v_{4}$, respectively, such that, $x_{1}^{\prime}$ and $x_{2}^{\prime}$, the new locations of $x_{1}$ and $x_{2}$, satisfy that $d\left(v_{1}, x_{1}^{\prime}\right)=d\left(v_{3}, x_{2}\right)$ and $d\left(v_{3}, x_{2}^{\prime}\right)=d\left(v_{1}, x_{1}\right)$. This movement induces the following change in the objective function:

$$
\left(d\left(v_{1}, x_{1}\right)-d\left(v_{3}, x_{2}\right)\right)\left(\lambda_{4}-\lambda_{5}\right)<0,
$$

what contradicts the optimality of $X_{p}$.

(ii) Since $\sigma_{3} \leqslant 3, \sigma_{5} \leqslant 3$ and $\sigma_{2}=4, X_{p}$ must satisfy that $d\left(v_{2}, x_{1}\right) \geqslant d\left(v_{3}, x_{2}\right)$ and $d\left(v_{2}, x_{1}\right) \geqslant d\left(v_{5}\right.$, $\left.x_{3}\right)$. In addition, we have by construction that $d\left(v_{2}, x_{1}\right) \leqslant 2$ then $d\left(v_{3}, x_{2}\right) \leqslant 2$ and $d\left(v_{5}, x_{3}\right) \leqslant 2$. This allows us to use the same arguments of Case 2(i) to prove that $d\left(v_{3}, x_{2}\right) \geqslant d\left(v_{5}, x_{3}\right)$ because $\lambda_{5}>\lambda_{6}$.

Therefore, $\lambda_{1}$ must be assigned to $v_{1}, \lambda_{2}$ to $v_{5}$ and $\lambda_{3}$ to $v_{3}$. In addition, since $\lambda_{1}, \lambda_{2}, \lambda_{3}, \lambda_{4}, \lambda_{5}, \lambda_{6}$ have been already assigned and $\lambda_{8}$ is assigned to $v_{8}$; Remark 2.2(i) implies that $\lambda_{7}$ is assigned to $v_{7}$. Repeating this argument for any $i>4$ we have that $\lambda_{2 i-1}$ is assigned to $v_{2 i-1}$. Thus, $\sigma_{2 i-1}=2 i-1$ and $\sigma_{2 i}=2 i$ for any $i=4, \ldots, p$.

This assignment of the $\lambda$-weights implies that $d\left(v_{2 i}, x_{i}\right)=d\left(v_{2 i+1}, x_{i+1}\right)$ for any $i=3, \ldots, p-1$. Indeed, since $\sigma_{2 i}=2 i<2 i+1=\sigma_{2 i+1}$ then $d\left(v_{2 i}, x_{i}\right) \leqslant d\left(v_{2 i+1}, x_{i+1}\right)$, and since $\lambda_{2 i+1}>\lambda_{2 i+2}$ we deduce that $x_{i+1}$ is located as close as possible 

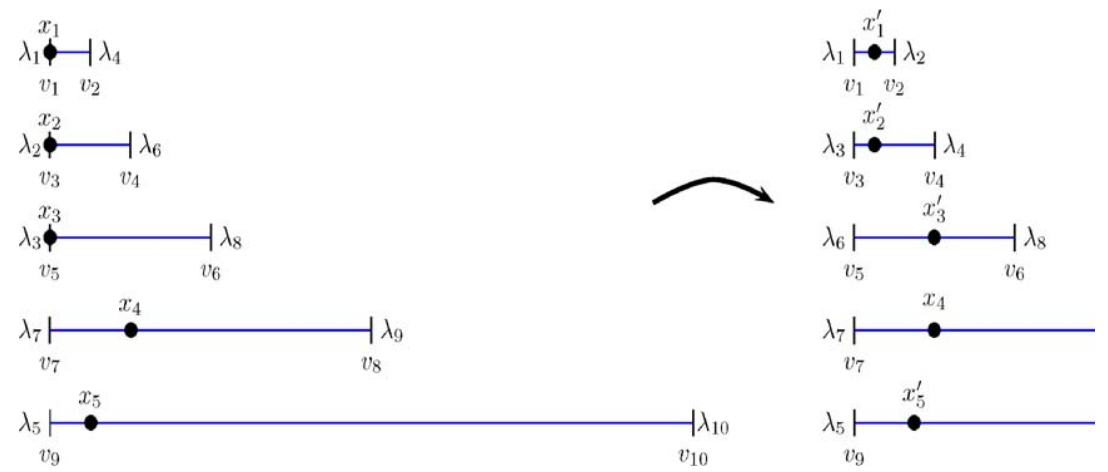

Fig. 5. Illustration of Case 4.

1 to $v_{2 i+1}, i=3, \ldots, p-1$. Hence, it implies that $d\left(v_{2 i}, x_{i}\right)=d\left(v_{2 i+1}, x_{i+1}\right)$ for any $i=3, \ldots, p-1$.

Moreover, with this assignment of the $\lambda$-weights and since, by (6), $2 \lambda_{2}>\lambda_{4}+\lambda_{5}+\lambda_{8}$ then the optimal location for $x_{1}, x_{2}$ and $x_{3}$ must be: $x_{1}=v_{1}, x_{2}=v_{3}$, $x_{3}=v_{5}$.

However, this is a contradiction because we will prove that the above configuration of $X_{p}$ does not

21 We prove that this amount is negative. Indeed, by the definition of the $\lambda$-weights, see (2) and (4)-(6), they provide an optimal solution of Problem (3). Indeed, move $x_{1}, x_{2}$ and $x_{3}$ to the new positions $x_{1}^{\prime}, x_{2}^{\prime}$ and $x_{3}^{\prime}$, respectively, where $x_{1}^{\prime}=v_{1}+1, x_{2}^{\prime}=v_{3}+1$, and $x_{3}^{\prime}=v_{5}+$ 3. Using the condition $d\left(v_{2 i}, x_{i}\right)=d\left(v_{2 i+1}, x_{i+1}\right)$ for any $i=3, \ldots, p$, this movement allows us to displace three units length: (1) $x_{i}$ towards $v_{2 i-1}$ for any even index $i=4, \ldots, p$ and (2) $x_{j}$ towards $v_{2 j}$ for any odd index $j=4, \ldots, p$; without any reassignment of the $\lambda$-weights corresponding to these nodes. Therefore, these movements produce the following change in the objective function:

$$
\begin{aligned}
& +\lambda_{1}+\lambda_{2}+\lambda_{3}+\lambda_{4}-\lambda_{5}-3 \lambda_{6}-3\left(\lambda_{7}-\lambda_{8}\right) \\
& +3\left(\lambda_{9}-\lambda_{10}\right)-3\left(\lambda_{11}-\lambda_{12}\right)+\ldots
\end{aligned}
$$

satisfy that $+\lambda_{1}+\lambda_{2}+\lambda_{3}+\lambda_{4}-\lambda_{5}-3 \lambda_{6}$ is negative. Besides,

$-3\left(\lambda_{7}-\lambda_{8}\right)+3\left(\lambda_{9}-\lambda_{10}\right)-3\left(\lambda_{11}-\lambda_{12}\right)+\ldots$

is negative because the sequence $\lambda_{7}-\lambda_{8}, \lambda_{9}-\lambda_{10}, \ldots$ is decreasing. This fact contradicts the optimality of $X_{p}$ since the objective function decreases.

Case 3: $j_{o}=4$. The proof is similar to the one in Case 2, and therefore it is omitted.
Case 4: $j_{o}>4$ (see Figs. 5 and 6).

Using Remark 2.2(ii), Lemma A.1 and a similar argument to that used in Case 2(i), $\lambda_{1}$ must be assigned to $v_{1}, \lambda_{2}$ to $v_{3}, \lambda_{2 i+2}$ to $v_{2 i}$ for $i=1, \ldots, j_{o}-2$, $\lambda_{2 i-3}$ to $v_{2 i-1}$ for $i=3, \ldots, j_{o}-2, \lambda_{2 j_{o}-3}$ to $v_{2 j_{o}-3}$, $\lambda_{2 j_{o}-5}$ to $v_{2 j_{o}-1}, \lambda_{2 j_{o}-1}$ to $v_{2 j_{o}-2}$, and $\lambda_{i}$ to $v_{i}$ for $i=2 j_{o}, \ldots, 2 p$.

Moreover, notice that, $\lambda_{\sigma_{2 i-1}}>\lambda_{\sigma_{2 i}}$ for $i>1$. Hence, following a similar argument to the one in Case 2, we obtain that $d\left(v_{2 i}, x_{i}\right)=d\left(v_{2 j-1}, x_{j}\right)$ whenever $\sigma_{2 j-1}-\sigma_{2 i}=1$.

For this assignment of the $\lambda$-weights, using (2) and (4)-(6), the optimal location of $x_{1}, x_{2}, x_{3}, x_{4}$ and $x_{5}$ must be $x_{1}=v_{1}, x_{2}=v_{3}, x_{3}=v_{5}$, and either

1. $x_{4}=v_{7}+4$ and $x_{5}=v_{9}+2$, if $j_{o}=5$ (Fig. 5)

or

2. $x_{4}=v_{7}+2$ and $x_{5}=v_{9}+4$, if $j_{o}>5$ (Fig. 6).

However, this is a contradiction because we will prove that the above configuration of $X_{p}$ does not provide an optimal solution of Problem (3). Move $x_{1}$, $x_{2}, x_{3}, x_{4}$ and $x_{5}$ to the new positions $x_{1}^{\prime}, x_{2}^{\prime}, x_{3}^{\prime}, x_{4}^{\prime}$ and $x_{5}^{\prime}$, respectively, where $x_{1}^{\prime}=v_{1}+1, x_{2}^{\prime}=v_{3}+1$, $x_{3}^{\prime}=v_{5}+4$, and

1. $x_{4}^{\prime}=v_{7}+4$ and $x_{5}^{\prime}=v_{9}+3$, if $j_{o}=5$ (Fig. 5).

2. $x_{4}^{\prime}=v_{7}+3$ and $x_{5}^{\prime}=v_{9}+4$, if $j_{o}>5$ (Fig. 6).

These displacements permit us to move $x_{i}$ towards either $v_{2 i-1}$ or $v_{2 i}$ for $i=6, \ldots, p$ without any re- 


\section{ARTICLE IN PRESS}
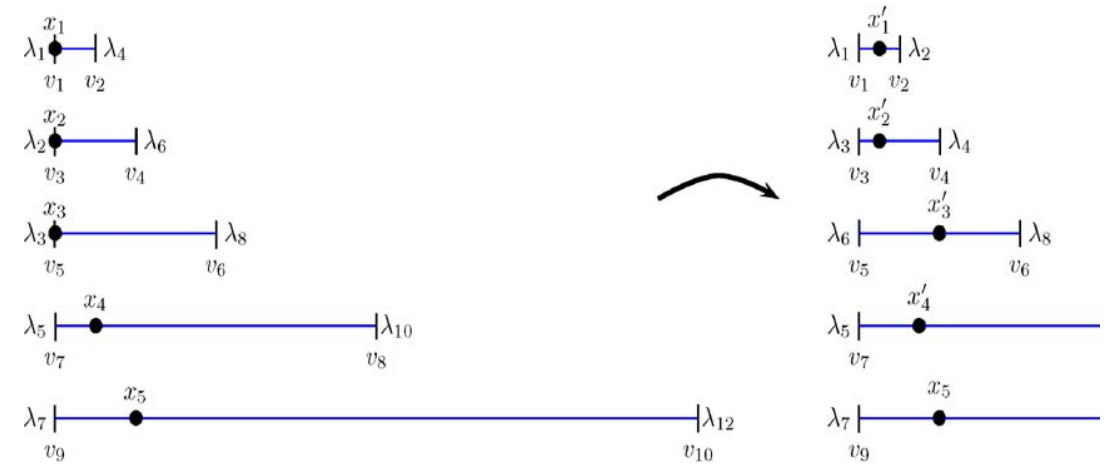

Fig. 6. Illustration of Case 4.

1 assignment of their corresponding $\lambda$-weights. (This is possible using the condition $d\left(v_{2 i}, x_{i}^{\prime}\right)=d\left(v_{2 j-1}, x_{j}^{\prime}\right)$

when $\sigma_{2 j-1}-\sigma_{2 i}=1$.) The change of the objective function is as follows:

(i) If $j_{o}=5$

$$
\begin{gathered}
+\lambda_{1}+\lambda_{2}+\lambda_{3}+\lambda_{4}+\lambda_{5}-4 \lambda_{8}-\lambda_{10}-\left(\lambda_{11}\right. \\
\left.-\lambda_{12}\right)+\left(\lambda_{13}-\lambda_{14}\right)-\left(\lambda_{15}-\lambda_{16}\right)+\ldots
\end{gathered}
$$

7 By (2) and (4)-(6), we have that $+\lambda_{1}+\lambda_{2}+\lambda_{3}+\lambda_{4}+$ $\lambda_{5}-4 \lambda_{8}-\lambda_{10}$ is negative (by the definition of the $\lambda$ -

9 weights $)$. Besides, $-\left(\lambda_{11}-\lambda_{12}\right)+\left(\lambda_{13}-\lambda_{14}\right)-\left(\lambda_{15}-\right.$ $\left.\lambda_{16}\right)+\ldots$ is negative because the sequence $\lambda_{11}-\lambda_{12}$, $11 \lambda_{13}-\lambda_{14}, \lambda_{15}-\lambda_{16}, \ldots$ is decreasing.

(ii) If $j_{o}>5$

$$
\begin{aligned}
& +\lambda_{1}+\lambda_{2}+\lambda_{3}+\lambda_{4}+\lambda_{5}-4 \lambda_{8}-\lambda_{10} \\
& +\sum_{\left\{j \geqslant 0,7+6 j<2 j_{o}-3\right\}}(-1)^{j+1} \cdot 0 \cdot\left(\lambda_{7+6 j}-\lambda_{12+6 j}\right)
\end{aligned}
$$

$$
\begin{aligned}
& +\sum_{\left\{j \geqslant 0,9+6 j<2 j_{o}-3\right\}}(-1)^{j+1} 4\left(\lambda_{9+6 j}-\lambda_{14+6 j}\right) \\
& +\sum_{\left\{j \geqslant 0,11+6 j<2 j_{o}-3\right\}}(-1)^{j+1}\left(\lambda_{11+6 j}-\lambda_{16+6 j}\right)
\end{aligned}
$$

15

$$
+r(-1)^{j_{r}}\left(\lambda_{2 j_{o}-3}-\lambda_{2 j_{o}-1}\right)+\sum_{j=j_{o}}^{p-1} t(-1)^{j_{t}+\left(j-j_{o}\right)}
$$

where

$t= \begin{cases}0, & \text { if } \exists j_{t} \text { such that } 2 j_{o}-3=7+6 j_{t}, \\ 4, & \text { if } \exists j_{t} \text { such that } 2 j_{o}-3=9+6 j_{t}, \\ 1, & \text { if } \exists j_{t} \text { such that } 2 j_{o}-3=11+6 j_{t} .\end{cases}$

$r= \begin{cases}0, & \text { if } \exists j_{r} \text { such that } 2 j_{o}+1=7+6 j_{r}, \\ 4, & \text { if } \exists j_{r} \text { such that } 2 j_{o}+1=9+6 j_{r}, \\ 1, & \text { if } \exists j_{r} \text { such that } 2 j_{o}+1=11+6 j_{r} .\end{cases}$

In case (i) we proved that $+\lambda_{1}+\lambda_{2}+\lambda_{3}+\lambda_{4}+$ $\lambda_{5}-4 \lambda_{8}-\lambda_{10}$ is negative. Moreover,

$$
\begin{aligned}
& +\sum_{\left\{j \geqslant 0,9+6 j<2 j_{o}-3\right\}}(-1)^{j+1} 4\left(\lambda_{9+6 j}-\lambda_{14+6 j}\right) \\
& +\sum_{\left\{j \geqslant 0,11+6 j<2 j_{o}-3\right\}}(-1)^{j+1}\left(\lambda_{11+6 j}-\lambda_{16+6 j}\right) \\
& +r(-1)^{j_{r}}\left(\lambda_{2 j_{o}-3}-\lambda_{2 j_{o}-1}\right) \\
& +\sum_{j=j_{o}}^{p-1} t(-1)^{j_{t}+\left(j-j_{o}\right)}\left(\lambda_{2 j+1}-\lambda_{2 j+2}\right)
\end{aligned}
$$

is negative because we can decompose the expression above in different sums, where each one of them constitutes a decreasing sequence in absolute value with alternate signs and being its first element negative.

Since in all the possible cases we get a contradiction, the initial hypothesis that $\lambda_{2}$ is assigned to a vertex with odd index is inconsistent. Therefore, using Lemma A. 1 we conclude that $\lambda_{2}$ can only be assigned to $v_{2}$. 


\section{References}

[1] M.O. Ball, T.L. Magnanti, C.L. Monma, G.L. Nemhauser (Eds.), Network Models, Handbooks in Operations Research and Management Science, vol. 7, North-Holland, Amsterdam; 1995.

[2] N. Boland, P. Domíguez-Marín, S. Nickel, J. Puerto, Exact procedures for solving the discrete ordered median problem, Ber. Fraunhofer ITWN 47 (2003).

Heuristic procedures for solving the discrete ordered median problem, Ber. Fraunhofer ITWN 46 (2003).

[4] Z. Drezner, H.W. Hamacher (Eds.), Facility Location, Applications and Theory, Springer, New York, 2002.

[5] R.L. Francis, T.J. Lowe, A. Tamir, Aggregation error bounds for a class of location models, Oper. Res. 48 (2) (2000) 294 $-307$.

[6] R.L. Francis, T.J. Lowe, A. Tamir, Worst-case incremental analysis for a class of $p$-facility location problems, Networks 39 (3) (2002) 139-143.

[7] S.L. Hakimi, Optimum locations of switching centers and the absolute centers and medians of a graph, Oper. Res. 12 (1964) 450-459.

[8] G.Y. Handler, P.B. Mirchandani, Location on Networks Theory and Algorithms, vol. 18, The MIT Press, Cambridge, 1979.

[9] J.N. Hooker, R.S. Garfinkel, C.K. Chen, Finite dominating sets for network location problems, Oper. Res. 39 (1) (1991) $100-118$.

[10] J. Kalcsics, S. Nickel, J. Puerto, A. Tamir, Algorithmic results for ordered median problems, Oper. Res. Lett. 30 (3) (2002) $149-158$.
[11] J. Kalcsics, S. Nickel, J. Puerto, Multifacility ordered median problems on networks: a further analysis, Networks 41 (1) (2003) 1-12.

[12] P.B. Mirchandani, R.L. Francis (Eds.), Discrete Location Theory, Wiley-Interscience Series in Discrete Mathematics and Optimization, Wiley, New York, 1990.

[13] S. Nickel, J. Puerto, A unified approach to network location problems, Networks 34 (4) (1999) 283-290.

[14] W. Ogryczak, On the distribution approach to location problems, Comput. Ind. Eng. 37 (1999) 595-612.

[15] J. Puerto, F.R. Fernández, The symmetrical single facility location problem, Preprint of the Mathematics Faculty, Universidad de Sevilla, 1995. symmetrical single facility location problem, J. Nonlinear Convex Anal. 1 (3) (2000) 321-342.

[17] J. Puerto, A.M. Rodríguez-Chía, F. Fernández-Palacín, Ordered Weber problems with attraction and repulsion, Stud. Locational Anal. 11 (1997) 127-141.

[18] A.M. Rodríguez-Chía, S. Nickel, J. Puerto, F.R. Fernández, A flexible approach to location problems, Math. Methods Oper. Res. 51 (1) (2000) 69-89.

[19] A.M. Rodríguez-Chía, J. Puerto, D. Pérez-Brito, J.A. Moreno, The $p$-facility ordered median problem on networks, Working paper, 2003.

[20] A. Tamir, An $O\left(p n^{2}\right)$ algorithm for the $p$-median and related problems on tree graphs, Oper. Res. Lett. 19 (1996) 59-64.

[21] R. Yager, On ordered weighted averaging aggregation operators in multicriteria decision making, IEEE Trans. Systems Man Cybernet. 18 (1988) 183-190. 